\title{
In-vessel colorimetry of Wendelstein 7-X first wall components: Variation of layer deposition distribution in OP1.2a and OP1.2b
}

\author{
G. Motojima ${ }^{1,2}$, S. Masuzaki ${ }^{1}$, C.P. Dhard ${ }^{3}$, M. Krause ${ }^{3}$, D. Naujoks ${ }^{3}$, Y. Hayashi ${ }^{1}$, S. Brezinsek ${ }^{4}$ and \\ the W7-X Team ${ }^{3}$
}

${ }^{1}$ National Institute for Fusion Science, National Institutes of Natural Sciences, 322-6 Oroshi-cho, Toki, Gifu 5095292, Japan

${ }^{2}$ SOKENDAI (The Graduate University for Advanced Studies), 322-6 Oroshi-cho, Toki, Gifu 509-5292, Japan

${ }^{3}$ Max-Planck Institute für Plasmaphysik, Wendelsteinstrasse 1, 17491 Greifswald, Germany

${ }^{4}$ Forschungszentrum Jülich, Institut für Energie und Klimaforschung - Plasmaphysik, 52425 Jülich, Germany

Email: motojima.gen@nifs.ac.jp

\begin{abstract}
A colorimetry study of the first wall panels in the Wendelstein 7-X (W7-X) was carried out using a compact color analyzer after Operation Phases OP1.2a and OP1.2b, in which graphite test divertor units were used, to estimate the wide-range distribution of the deposition layer. The color analyzer was used to measure the intensities of the Red, Green and Blue (RGB) channels, which correspond to reflection rates, for all first-wall panels after OP1.2a and OP1.2b. A significant difference in the RGB values was found between OP1.2a and OP1.2b. The color pattern on the panels was roughly the same for all the five toroidal modules of W7-X. The deposition layer thickness was estimated from the RGB values using a single-layer model. A thin average deposition layer $(10 \pm 6 \mathrm{~nm})$ was estimated for OP1.2a. On the other hand, a thicker average deposition layer $(25 \pm 8 \mathrm{~nm})$ was estimated for OP1.2b.
\end{abstract}

\section{Introduction}

Fueling particle control is one of the most important issues in fusion machines. For the particle control, in addition to the fueling methods and exhaust vacuum pumping system, fueling retention in the plasma-facing components (e.g., first wall) is critical. Fuel retention depends on properties and the conditions of the first wall, such as material type and its temperature. It has been reported that the retention of the fuel particles in a metal wall is one-tenth smaller than in a carbon wall [1]. In the Large Helical Device (LHD) [2], global particle balance analysis has shown dynamic wall retention of fueling particles (helium) in long pulse discharges (48 minutes) [3]. A deposition layer, mainly composed of carbon, forms on plasma-facing components of carbon-dominated devices and may contribute to wall retention as a co-deposition [4]. To verify this hypothesis, it is important to understand the absorption mechanism of the fuel by the deposition layer and to quantitatively evaluate the deposition layer. Specimen analysis is commonly used to evaluate the thickness and the microscopic structure of a deposition layer. 
However, it is difficult to obtain a finite number of specimens that cover the entire area of interest in a large fusion device. Furthermore, the analysis of specimens is time-consuming. Accordingly, color analysis was conducted for ASDEX-U [5] and TEXTOR-94 [6]. However, the measurement area was still limited and thus a wide area of the vacuum vessel was not included.

Color measurement has been applied as a simple method for obtaining the deposition layer thickness in the LHD [7]. Colorimetry, which has been used for QUEST [8], can be used to obtain the wide-range distribution of the deposition layer. Such an analysis allows quantitative evaluation of the fuel retention [9]. In the present study, colorimetry is applied to the first wall of Wendelstein 7-X (W7-X) [10] after the experimental campaigns of Operation Phases 1.2a (OP1.2a) and 1.2b (OP1.2b) [11]. W7-X started operation in 2015. Plasma experiments with graphite Test Divertor Units (TDUs) were conducted in OP1.2a and OP1.2b. In W7-X, erosion and deposition were investigated using graphite coated TZM (Ti-Zr-Mo)-screws after both operation phases [12] and the first-wall components were inspected after OP1.2b [13]. In addition, the distribution of the deposition layer on the first wall panels from the early phase of an experimental campaign is useful because the history of the deposition layer formation is important for wall retention physics. The present study reports the colorimetry results for W7-X.

The rest of this paper is organized as follows. In section 2, the process used for evaluating the deposition layer thickness, the color analyzer and the single-layer model are described. In section 3, the colorimetry results are presented and used to estimate the deposition layer thickness. Conclusions are given in section 4.

\section{Experimental set-up}

\subsection{Process used for evaluating thickness distribution of deposition layer}

The deposition layer thickness was evaluated using the three steps. In the first step, Red, Green and Blue (RGB) values, which correspond to the reflection intensities at specific wavelengths, were measured using the color analyzer. Here, the investigated spot is illuminated with white LED light. In the spectrum for the white LED, a large peak occurs in the blue region and there is a broad spectral power distribution in red and green region. In the second step, the RGB values were converted to a reflection rate using a linear relation. Ellipsometric measurements, which measured surface reflection rate, of specimens irradiated by LHD plasmas showed a linear relation between RGB values and the reflection rate [7]. Therefore, a linear relation was adopted in this study. In the third step, the reflection rate was converted to a deposition layer thickness. Here, we assume a single-layer model [14]. This model considers the deposition thickness to be a function of the reflection rate (see section 2.3 for details). The compatibility of the single-layer model has been confirmed via a composition with specimen analysis results for the LHD [7]. The material composition of the plasma-facing components in W7-X is similar to that in the LHD. In both machines, the first wall panel is composed of stainless steel and the divertor is composed of graphite. Therefore, the single-layer model should be suitable for the first wall panels in W7-X. Wall conditionings such as boronization probably affect the composition of the deposition layer. However, this effect is not taken into account in this study.

\subsection{Reflection rate measurement using compact color analyzer}


Figure 1 shows photographs of the color analyzer utilized in this study and its application to a first wall panel. The 'DM-1' color analyzer, produced by Hitachi Kinzoku Corporation [15], was used. The internal structure of the integrating sphere inside the analyzer is shown in Fig. 2. The light emitted from the white LED is injected into the target as homogeneous standard light by the diffusion plate. The light reflected from the target, including the diffusion light, is captured by the photodiode sensor, which has peak intensities of red, green and blue of 615,540 and $465 \mathrm{~nm}$, respectively. The intensities of the three specific wavelengths are displayed on a monitor. The analyzer can also measure the hue, saturation and brightness values. Before measurements, the color analyzer was calibrated using a standard white with a reflection rate of 0.73 , as measured using ellipsometry. The full specifications of the color can be found elsewhere [7].

\subsection{Single-layer model}

This section describes the single-layer model used to convert the reflection rate to a deposition layer thickness. The simple three-phased model (atmosphere, deposition layer and substrate area) shown in Fig. 3(a) is assumed. With this model, the reflection coefficient of light, $R$, can be described as follows [14]:

$r_{i j}=\frac{n_{i} \cos \theta_{i}-n_{j} \cos \theta_{j}}{n_{i} \cos \theta_{i}+n_{j} \cos \theta_{j}}$

$r=\frac{r_{12}+r_{23} e^{2 i \varnothing}}{1+r_{23} r_{12} e^{2 i \phi}}$

$\emptyset=\frac{2 \pi n_{2} d \cos \theta_{2}}{\lambda}$

$R=|r|^{2}$,

where $r_{i j}$ is the Fresnel reflection coefficient at the boundary between $i$ and $j$ and $r$ are the overall electric field of the light and the phase factor, respectively, $d$ is the deposition layer thickness and $n$ is the complex reflective index. The reflection rate is a function of the deposition layer thickness.

In this study, the complex reflective index was calculated as $n+\mathrm{i} k$, where $n$ and $k$ are the real and imaginary parts, respectively. For the deposition layer, $n=1.6$ and $k=0.2$, and for the substrate of the first wall panels (stainless steel), $n=1.5$ and $k=2.9$. The complex reflective index for the deposition layer was preliminarily measured using ellipsometry with a panel of the inner divertor closure [16]. The complex reflective index for the first wall panel is unknown and thus assumed to be the same as that used in the LHD. The single layer model shows that $k$ dependence on the absolute value of the thickness is rather weak, but $n$ dependence is strong. For hydrogen, $n \sim 1$ [17], and for boron, $n \sim 3$ [18]. Therefore, hydrogen content of the layers might increase the thickness evaluation and boron content of the layers might reduce the thickness evaluation. Thus, influence of the hydrogen and boron content of the layers on optical layer properties should be taken into account. However, the quantitative effect remains unclear and will be clarified by ellipsometric measurements in future. The composition of the first wall material in W7-X is the same as that in the LHD and thus it is reasonable to use the value for the latter in this study. Figure 3(b) shows the relation between the estimated thickness and the reflection rate. A clear dependence of thickness on the reflection rate can be seen. In the thickness of over $100 \mathrm{~nm}$, the similar dependence of reflection rate on the thickness is expected again as a second-order. However, the 
thickness derived in this study corresponds to first-order assumption of the layer thickness of less than $100 \mathrm{~nm}$. The surface roughness might influence the reflection coefficient measurement. However, the effect of surface roughness on the visible wavelength is not appreciable if the roughness is sufficiently lower than the visible light wavelength.

\section{Experimental results of colorimetry for OP1.2a and OP1.2b}

\subsection{Colorimetry results}

The procedure used for the colorimetry measurements of the first wall panels was as follows. Eight measurement points were selected on each panel. They were roughly equally distributed in each first wall panel. The representative RGB value of each panel was determined by averaging the RGB values for the eight points. There are approximately 200 first wall panels in W7-X. More than 1,600 data points were thus used to measure the RGB values of all first wall panels.

We measured the RGB values for all the first wall panels after OP1.2a and OP1.2b. The RGB values were visualized as a color pattern. Each color is printed according to its RGB value. Figures 4(a) and (b) show the color patterns for OP1.2a and OP1.2b, respectively. These patterns are significantly different. A relatively dark color pattern was obtained for OP1.2b. Dark colors indicate small RGB values, which correspond to a small reflection rate. Each of the five W7-X modules is composed of two Half Modules (HMs). Each of the HMs contains almost an equal number of panels. However, the exact numbers are shown in figure 4 . In the following section, the RGB values, reflection rate and deposition layer thickness using the sorted first wall panels are discussed.

\subsection{RGB values for the first wall panels and estimation of deposition layer thickness in module 1}

Figure 5(a) shows the RGB values and reflection rates for the first wall panels in module 1. High RGB values and high reflection rates were obtained for OP1.2a, suggesting the formation of a thin deposition layer. However, for some panels, small reflection rates close to 0.5 were observed, suggesting the formation of localized deposition layers. Smaller reflection rates were obtained for OP1.2b compared to those of OP1.2a, suggesting that an additional deposition layer formed in OP1.2b. The panels with the smallest reflection rates for OP1.2a and OP1.2b were different. Therefore, the source of deposition layer formation might also be different.

The deposition layer thickness was estimated using the single-layer model. Figure 5(b) shows the estimated deposition layer thickness for OP1.2a and OP1.2b. As suggested by Figure 5(a), a thin deposition layer was estimated for OP1.2a. For the panels with small reflection rates, a thickness of approximately $20 \mathrm{~nm}$ was estimated. In contrast, for OP1.2b, a thicker deposition layer was estimated. Almost all first wall panels show thicker deposition layer formations for OP1.2b. The largest thickness was approximately $40 \mathrm{~nm}$. The thickness distribution patterns were different between OP1.2a and OP1.2b, suggesting that the formation of the deposition layers was different.

\subsection{Thickness distribution for all modules}

Figure 6 shows the estimated deposition layer thickness for all modules. For each module, a thicker deposition layer formed during OP1.2b compared to OP1.2a. The thickness distributions of the modules are roughly similar. Here, we discuss the reasons for the different formations of the deposition layers observed in OP1.2a and OP1.2b. 
Although the plasma-facing components were the same for OP1.2a and OP1.2b, the total plasma discharge time of OP1.2b was about 2.4 times corresponding time of OP1.2a (9054 s vs. $3776 \mathrm{~s}$ ). Moreover, in OP1.2b, the time of glow discharge was significantly lower and less erosion from the first wall is estimated [19]. Different plasma parameters, magnetic field configurations, divertor loads and boronization were conducted for the first time in OP1.2b, which might have impacted the formation of the deposition layer. Further analysis is thus required.

Figure 7 shows the ratio of the deposition layer thickness for OP1.2b to that for OP1.2a. For each module, the ratio increased in HM11, HM21, HM31, HM41 and HM51. This might be related to the asymmetric loads between upper and lower TDUs. However, the reason for this remains unclear and thus further analysis is required.

Figure 8 shows the average estimated thickness of the deposition layer of the panels for all modules. The average estimated thickness is $10 \pm 6 \mathrm{~nm}$ for OP1.2a and $25 \pm 8 \mathrm{~nm}$ for OP1.2b. Thus, the thickness of the deposition layer formed in $\mathrm{OP} 1.2 \mathrm{~b}$ is 2.5 times larger than that formed in OP1.2a which is comparable to 2.4 times longer plasma duration in OP1.2b. We also investigated the deposition layer thickness in each module. As shown in Fig. 8, the average thickness of the deposition layer in each module was also evaluated by averaging the RGB values obtained for the first wall panels in that module. Although the color pattern is roughly the same for all modules, there is a small variation in the thickness distribution. Module 3 had the largest deposit and module 2 had the smallest deposit. Of note, this tendency was observed for both OP1.2a and OP1.2b. If we assume that the density of the deposition layer is similar to fine grain graphite $\left(1.5 \mathrm{~g} / \mathrm{cm}^{3}\right)$ but not to graphite $\left(2.3 \mathrm{~g} / \mathrm{cm}^{3}\right)$, the total deposition amount can be estimated. Since the surface area of the first wall panels observed by the colorimetry is approximately $87 \mathrm{~m}^{2}$, the total deposition amount is $10 \mathrm{~nm} \times 87 \mathrm{~m}^{2} \times 1.5 \mathrm{~g} / \mathrm{cm}^{3}=1.3 \mathrm{~g}$ for OP1.2a and $25 \times 87 \mathrm{~m}^{2}$ $\times 1.5 \mathrm{~g} / \mathrm{cm}^{3}=3.2 \mathrm{~g}$ for OP1.2b. Here, these values are only correct with the assumption that the whole layer consists of carbon. These values can be compared with those obtained using global carbon balance analysis [20]. Besides the measured deposition $(<33 \mathrm{~nm})$ on the wall panels, a limited dust formation and flake delamination were observed on graphite plasma-facing component. However, the plasma performance was not affected at all due to the relatively small amount of released material produced via these mechanisms.

\section{Conclusion}

This study conducted colorimetry of the first wall components in W7-X obtained using a compact color analyzer after the experiment campaigns with the first divertor operation to evaluate the wide-range distribution of the deposition layer.

We measured the RGB values of all the first wall panels (more than 1,600 data points). The colorimetry results show a clear difference in RGB values between OP1.2a and OP1.2b. The color patterns were roughly similar for all modules. The deposition layer thickness was estimated using a single-layer model. Thin $(10 \pm 6 \mathrm{~nm})$ and thicker $(25 \pm 8 \mathrm{~nm}$ ) deposition layers were estimated for OP1.2a and OP1.2b, respectively. The thickness of the deposition layer formed during OP1.2b was 2.5 times larger than that formed during OP1.2a. The thickness especially increased in half modules HM11, HM21, HM31, HM41 and HM51 for OP1.2b. Although the relation between the reflection rate and the deposition layer thickness should be verified by micro-structure analysis, the colorimetry measurements can provide the global characteristics of the deposition layer, bridging the gap between postmortem analysis and global particle balance analysis. 


\section{Acknowledgments}

This study was supported by the NIFS Stellarator-Heliotron Association Committee (URSX209) and by a JSPS KAKENHI grant (18H01203). G.M. is grateful for the fruitful discussions regarding colorimetry with Dr. K. Matsumoto of Honda R \& D Co. Ltd. and with Dr. N. Yoshida of Kyushu University. This work has been carried out within the framework of the EUROfusion Consortium and has received funding from the Euratom research and training programme 2014-2018 and 2019-2020 under grant agreement No 633053. The views and opinions expressed herein do not necessarily reflect those of the European Commission.

\section{References}

[1] Brezinsek S et al, 2013, Fuel retention studies with the ITER-Like Wall in JET, Nucl. Fusion, 53, 083023.

[2] Takeiri Y et al., 2017, Extension of the operational regime of the LHD towards a deuterium experiment, Nucl. Fusion, 57, 102023.

[3] Motojima G et al., 2015, Global helium particle balance in LHD, J. Nucl. Mat., 463, 1080.

[4] Tokitani M et al., 2015, Plasma wall interaction in long-pulse helium discharge in LHD - Microscopic modification of the wall surface and its impact on particle balance and impurity generation, J. Nucl. Mat., 463, 91.

[5] Pugno R et al., 2009, Investigation of local carbon transport in the ASDEX Upgrade divertor using ${ }^{13} \mathrm{CH}_{4}$ puffing, J. Nucl. Mat., 390-391, 68.

[6] Wienhold P et al., 1997, Colorimetric measurements of carbon erosion and deposition rates on extended areas of plasma facing components in TEXTOR, J. Nucl. Mat., 241-243, 804.

[7] Motojima G et al., 2017, Wide-range evaluation of the deposition layer thickness distribution on the first wall by reflection coefficient measurements, Nucl. Mater. Energy, 12, 1219-1223.

[8] Wang Z et al., 2017, Measurement of thickness of film deposited on the plasma-facing wall in the QUEST tokamak by colorimetry, Review of Scientific Instruments, 88, 093502.

[9] Motojima G et al., IAEA FEC 2016 in Kyoto, Japan, Global particle balance and its relationship with the plasma wall interaction emerging in long pulse discharges on the large helical device, EX/P8-3.

https://nucleus.iaea.org/sites/fusionportal/Shared\%20Documents/FEC\%202016/fec2016preprints/preprint0516.pdf

[10] Sunn Pedersen T et al., 2017, Key results from the first plasma operation phase and outlook for future performance in Wendelstein 7-X, Phys. Plasmas, 24, 055503.

[11] Sunn Pedersen T et al., 2019, First results from divertor operation in Wendelstein 7-X, Plasma Phys. Control. Fusion, 61, 014035.

[12] Dhard C P et al, 2019, Erosion and deposition investigations on Wendelstein 7-X first wall components for the first phase in divertor configuration, Fusion Eng. Des. 146242.

[13] Dhard C P et al., 2019, Inspection of W7-X Plasma-Facing Components after the Operation Phase 1.2b: observations and first assessments, Physica Scripta, in press. 
[14] Born M and Wolf E, Principles of Optics $6^{\text {th }}$ Edition: Electromagnetic Theory of Propagation, Interference and Diffraction of Light. ISBN: 9781483103204

[15] Ono Y et al., 2019, Life Estimation of Rolling Bearings Based on the Colors on Sliding Surfaces, SAE Technical Paper 2019-01-0180.

https://doi.org/10.4271/2019-01-0180

[16] Dittmar T, private communication.

[17] Peck E R and Huang S, 1977, Refractivity and dispersion of hydrogen in the visible and near infrared, J. Opt. Soc. Am. 67, 1550-1554. https://doi.org/10.1364/JOSA.67.001550

[18] Morita N and Yamamoto A, 1975, Optical and Electrical Properties of Boron, Jpn. J. Appl. Phys. 14825.

[19] Brezinsek S et al, 2019, Introduction to TG Plasma-Wall Interaction Session, W7-X Workshop 2019, 14-17 May 2019 in Greifswald, Germany (private communication).

[20] Mayer M et al., 2019, Material erosion and deposition on the divertor of W7-X, Physica Scripta, in press. 
(a)
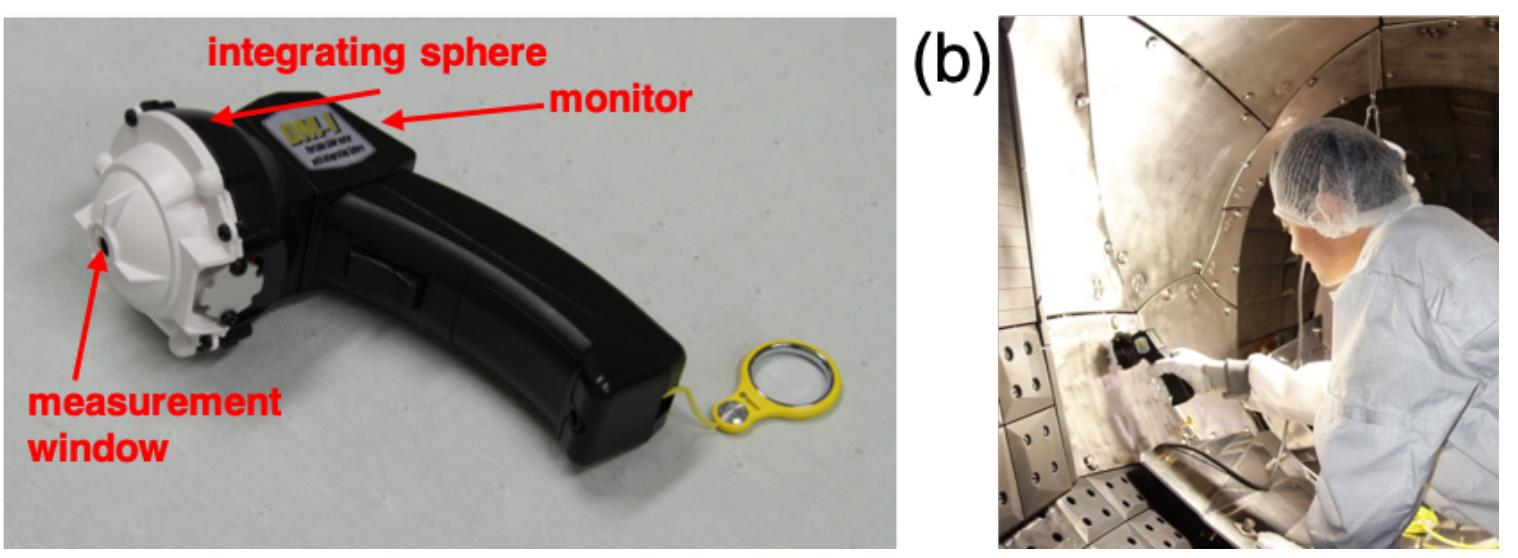

Fig 1. Photographs of (a) color analyzer and (b) its application to the first wall of W7-X. More than 1,600 points were measured in this study. 

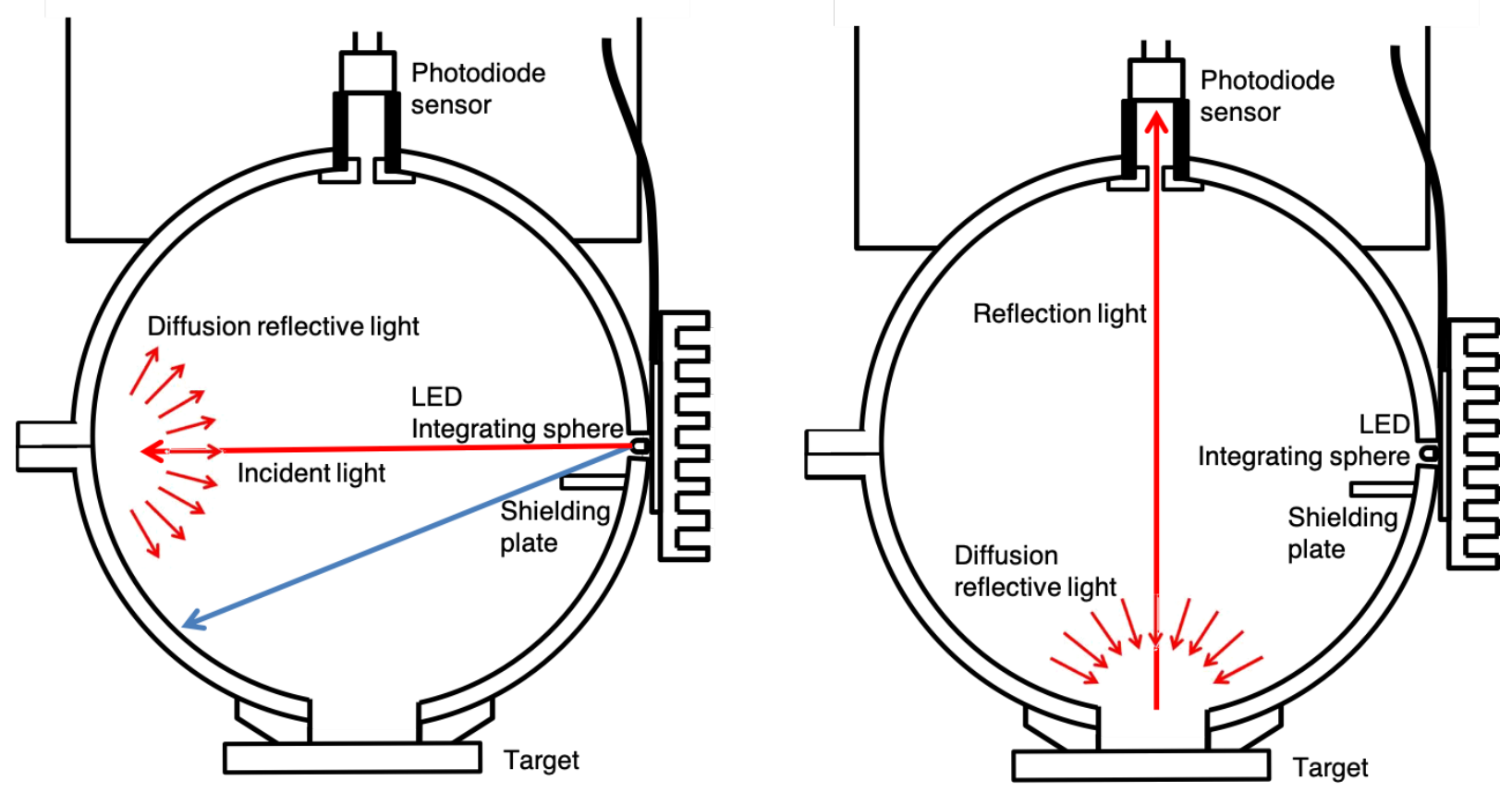

Fig. 2 Operation principle of colorimetry method. (a) Formation of incident light from the integrating sphere and (b) capture of light reflected from the target. 
(a)

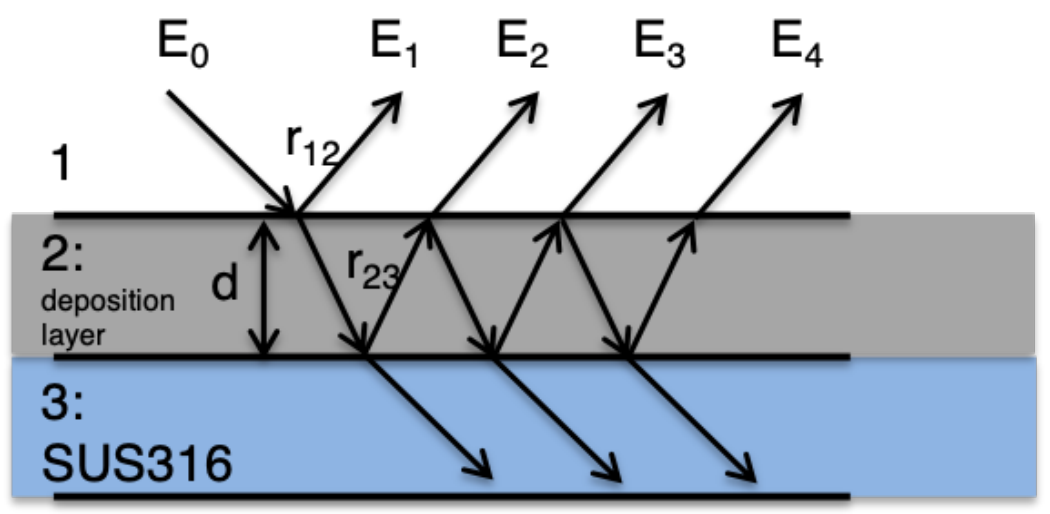

(b)

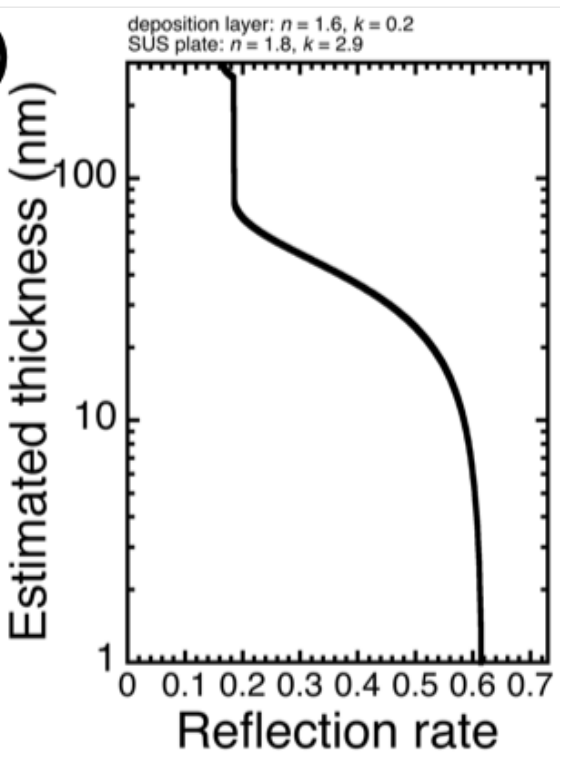

Fig. 3 (a) Three-phased model (atmosphere, deposition layer and substrate). $\theta$ is the angle of refraction at each boundary. (b) Estimated thickness as a function of reflection rate from the single-layer model. 


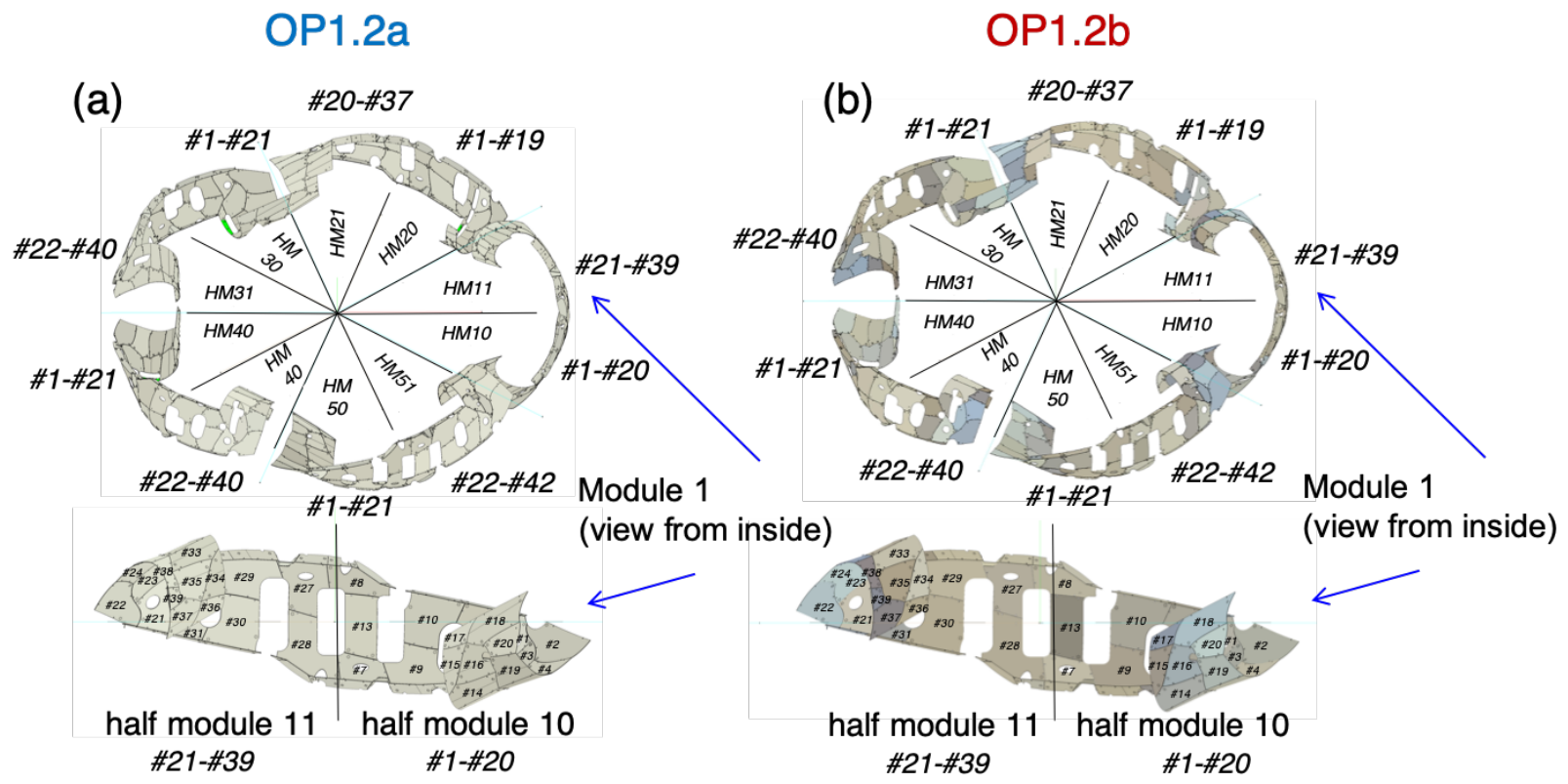

Fig. 4 Color pattern of first wall panels after (a) OP1.2a and (b) OP1.2b. W7-X has five modules. Each module is composed of two Half Modules (HMs). The panel numbers belonging to each HMs are shown in the figure i.e. \#1-\#20 are located in HM10. The panel numbers are indicated only in visible panels. 

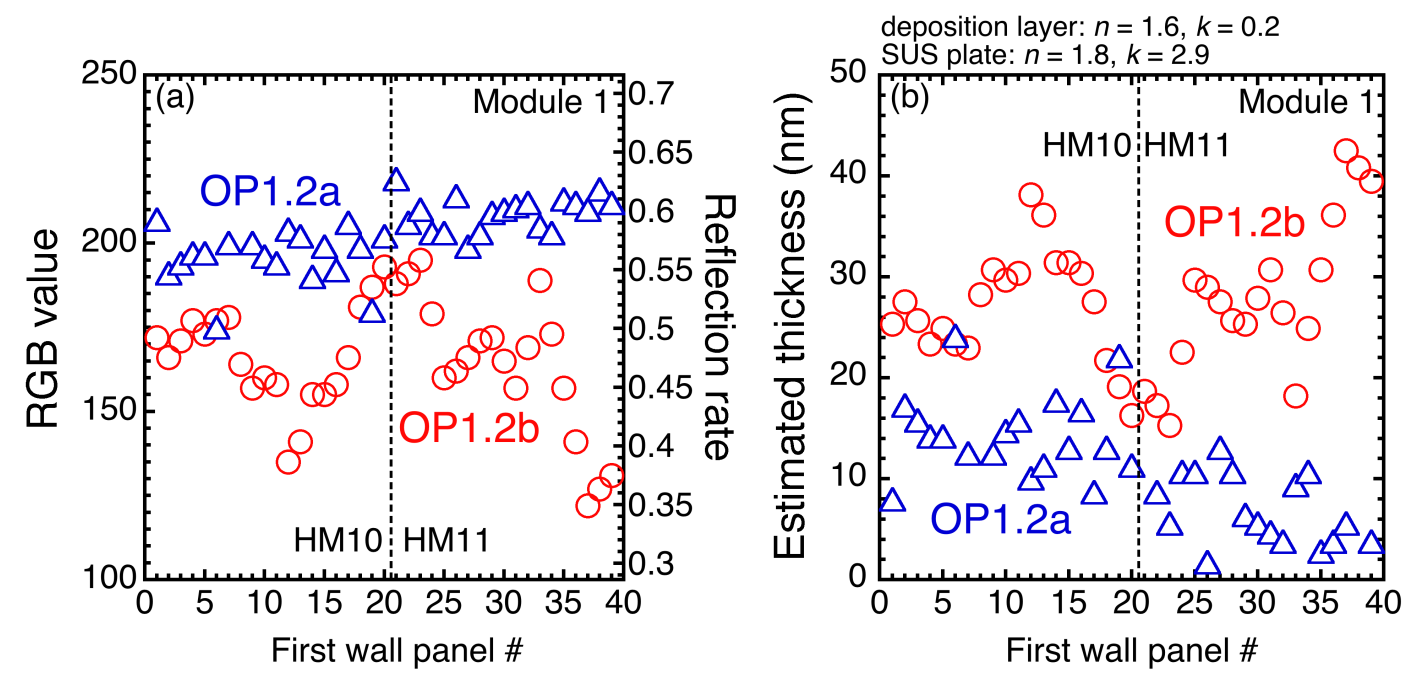

Fig. 5 (a) RGB values and the reflection rate of first wall panels in module 1 for OP1.2a and OP1.2b. (b) Estimated deposition layer thickness for OP1.2a and OP1.2b. In both these figures, triangles (blue) and circles (red) show the data points for OP1.2a and OP1.2b respectively. 


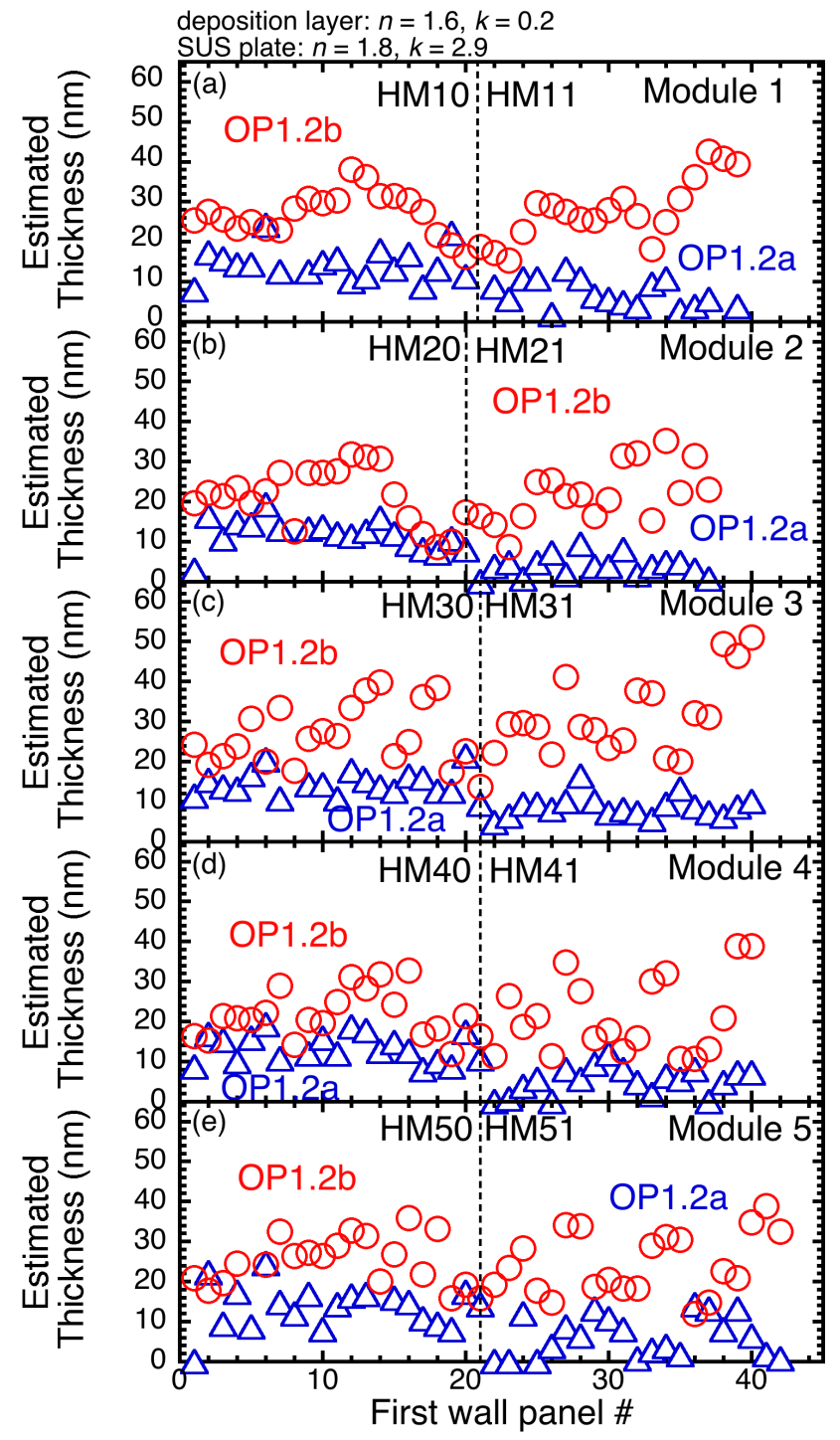

Fig. 6. Estimated deposition layer thickness of first wall panels in (a) module 1, (b) module 2, (c) module 3 , (d) module 4 and (e) module 5 . Triangles (blue) and circles (red) show the data points for OP1.2a and OP $1.2 \mathrm{~b}$ respectively. 


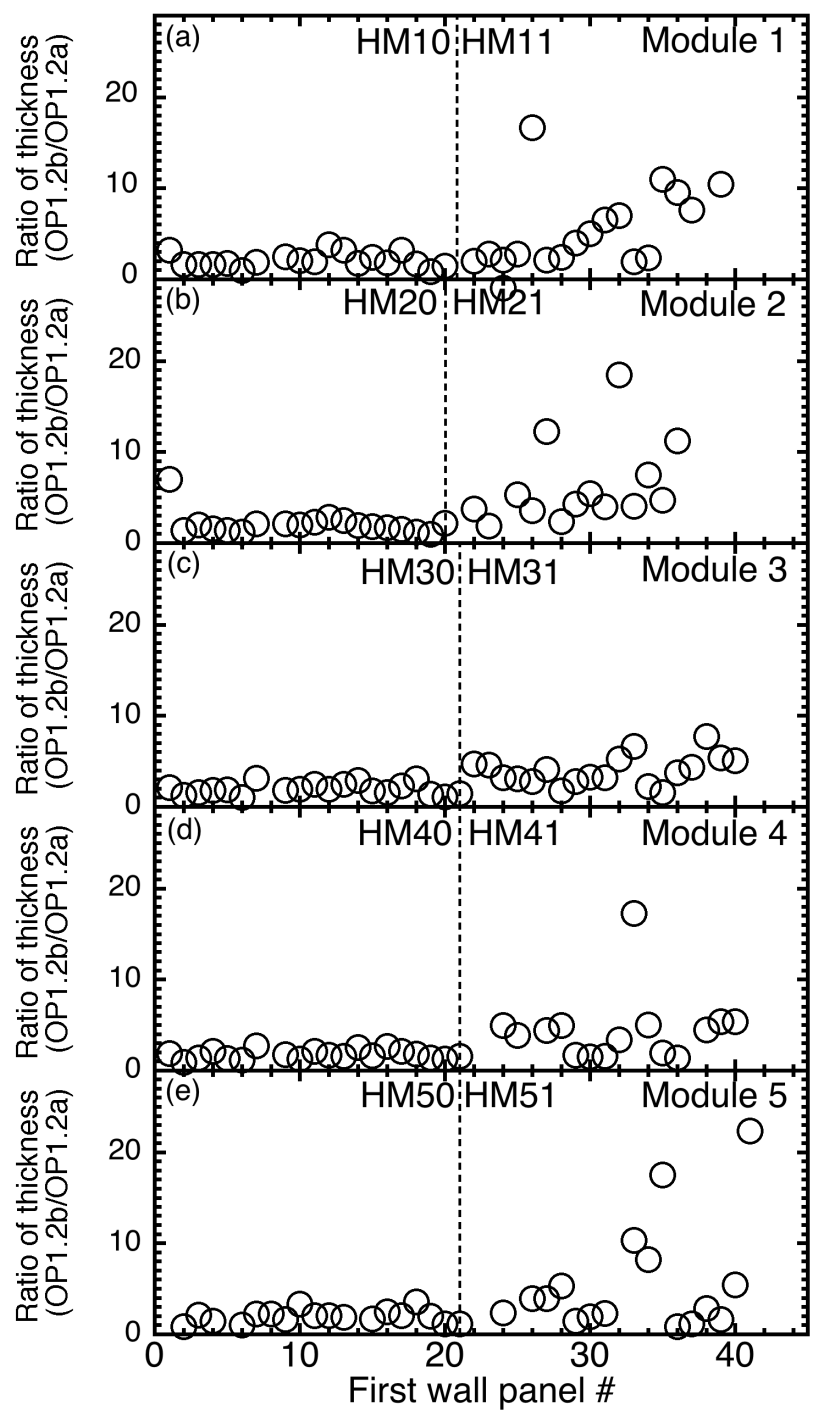

Fig. 7 Ratio of deposition layer thickness of OP1.2b to OP1.2a in (a) module 1, (b) module 2, (c) module 3, (d) module 4 and (e) module 5 . 


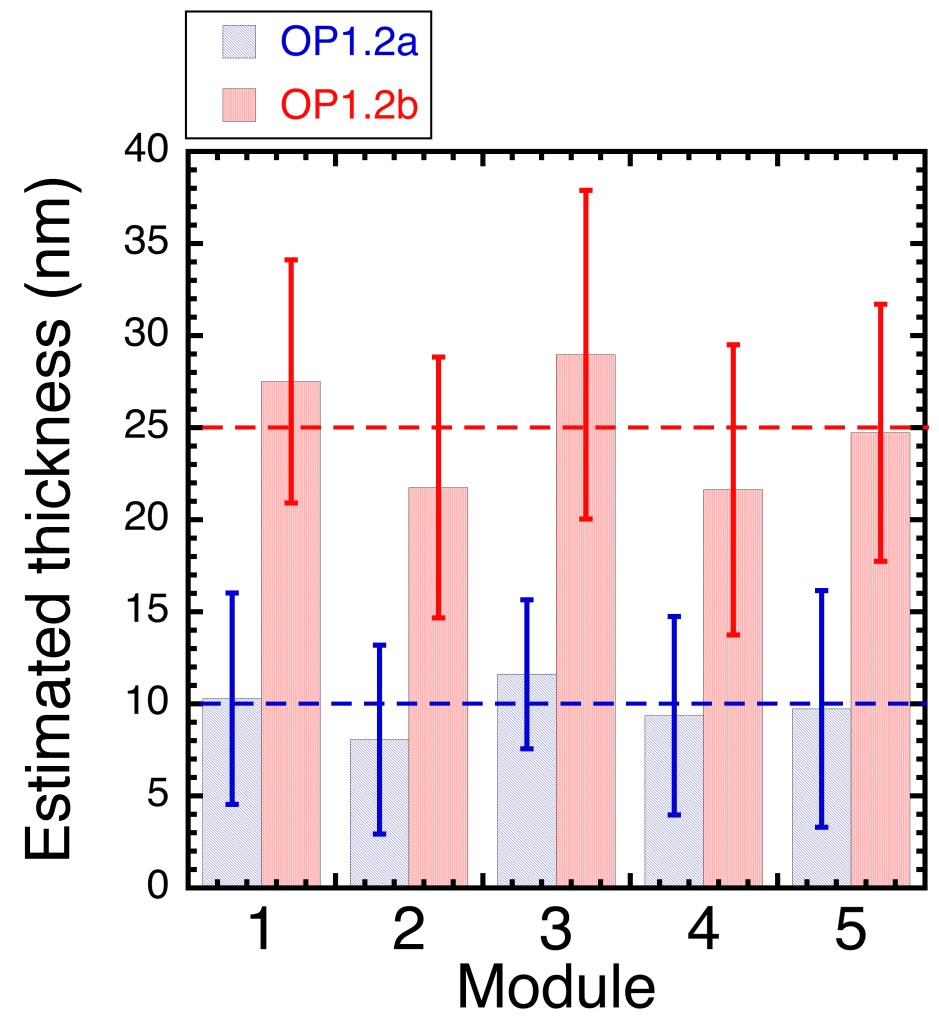

Fig. 8 Average deposition layer thickness for OP1.2a (blue) and OP1.2b (red). Blue and red dotted lines show average thickness of all first wall panels for OP1.2a and OP1.2b, respectively. Error bars indicate standard deviation of the estimated thickness in each module. 